\title{
MIXED FINITE ELEMENT FOR THE DYNAMIC ANALYSIS OF ORTHOTROPIC FLEXIBLE SHALLOW SHELLS
}

\author{
Leonid Yulianovich Stupishin ${ }^{1^{*}}$ Konstantin Evgenyevich Nikitin ${ }^{1}$ \\ ${ }^{1}$ Southwest State University, Kursk, Russia
}

\begin{abstract}
A finite-element methodology for studying the forced oscillations of orthotropic flexible shallow shells relative to the initial deformed state defined on the basis of a geometrically nonlinear deformation theory is proposed. To derive the finite-element equations, the Galerkin method is used in combination with the mixed formulation of the problem. The final finite-element equations have a simple structure and numerical integration is not required for calculating the matrices and vectors of finite elements. The accuracy and convergence of the mixed finite element is analyzed. Based on the developed methodology, the influence of geometric nonlinearity on the process of shell oscillations is studied.
\end{abstract}

Key words: Flexible shells, Orthotropy, Oscillations, Mixed finite element method, Galerkin method

\section{INTRODUCTION}

Finite element methodology for flexible shallow shells is proposed based on orthotropic material model. In the obtained finite-element equations it is possible to set independently of each other the tensile-compressive stiffness and the bending stiffness of the element. This makes it possible to use this finite element to calculate ribbed, multilayer and reinforced concrete shells after calculating their reduced characteristics.

The derivation of finite-element equations in mixed formulation is carried out using by the Galerkin method in the weak form [01-04]. This method allows to do without the construction procedure of the functional of problem and to solve equations of problem in the form in which they are written. Using mixed finite-element formulation, there is a decrease of the condition number of the matrix of the finite element and reducing the accumulation of rounding errors, compared with the finite-element in displacements formulation [05].

All this allows the use of linear shape functions to construct the finite element and to obtain the matrix and vectors of the finite element in explicit form. This allows us not to use numerical integration, which improves the accuracy of calculations.

\section{DESCRIPTION OF THE FINITE ELEMENT CALCULATION METHODOLOGY}

Equations of the forced oscillations of the orthotropic shell based on the quadratic nonlinear theory of shallow shells [01, 02] have a form:

$$
\begin{aligned}
& \frac{\partial}{\partial r}\left(C_{1}(r) \frac{\partial^{2} \varphi(r, t)}{\partial r^{2}}-\frac{C_{2}(r)}{r} \frac{\partial \varphi(r, t)}{\partial r}+r \frac{\partial}{\partial r}\left(C_{1}(r) \frac{\partial^{2} \varphi(r, t)}{\partial r^{2}}-\frac{C_{12}(r)}{r} \frac{\partial \varphi(r, t)}{\partial r}\right)+\right. \\
& \left.+C_{12}\left(\frac{\partial^{2} \varphi(r, t)}{\partial r^{2}}-\frac{1}{r} \frac{\partial \varphi(r, t)}{\partial r}\right)\right)+\frac{\partial}{\partial r}\left(\frac{\partial w(r, t)}{\partial r} \frac{\partial f(r)}{\partial r}\right)+\frac{1}{2} \frac{\partial}{\partial r}\left(\left(\frac{\partial w(r, t)}{\partial r}\right)^{2}\right)=0
\end{aligned}
$$

$$
\begin{aligned}
& \frac{1}{r} \frac{\partial}{\partial r}\left(D_{1}(r) \frac{\partial^{2} w(r, t)}{\partial r^{2}}+\frac{D_{2}(r)}{r} \frac{\partial w(r, t)}{\partial r}-\frac{\partial}{\partial r}\left(r D_{1}(r) \frac{\partial^{2} w(r, t)}{\partial r^{2}}+D_{12}(r) \frac{\partial w(r, t)}{\partial r}\right)\right)+ \\
& +\frac{1}{r} \frac{\partial}{\partial r}\left(\frac{\partial \varphi(r, t)}{\partial r} \frac{\partial f(r)}{\partial r}\right)+\frac{1}{r} \frac{\partial}{\partial r}\left(\frac{\partial \varphi(r, t)}{\partial r} \frac{\partial w(r)}{\partial r}\right)=\frac{h h}{g} \frac{\partial^{2} w(r)}{\partial t^{2}}-q(r, t)
\end{aligned}
$$

We introduce the dimensionless variables:

$$
\begin{aligned}
& \rho=r / \frac{r h}{\sqrt[4]{\mu}} ; \bar{t}=t \sqrt{\frac{g}{\gamma h^{3} C}} ; \Phi=-\frac{C \sqrt[4]{\mu^{3}}}{h} \frac{\partial \varphi(r, t)}{\partial r} ; \\
& \theta=\sqrt[4]{\mu} \frac{\partial w(r, t)}{\partial r} ; K=\frac{\sqrt{\mu}}{h} \frac{\partial f(\rho)}{\partial \rho} ; \\
& \bar{C}_{1}=\frac{C_{1}}{C} ; \bar{C}_{2}=\frac{C_{2}}{C} ; \bar{C}_{12}=\frac{C_{12}}{C} ; \\
& \bar{D}_{1}=\frac{C \mu}{h^{2}} D_{1} ; \bar{D}_{2}=\frac{C \mu}{h^{2}} D_{2} ; \bar{D}_{12}=\frac{C \mu}{h^{2}} D_{12} ; \\
& D_{12}=D_{2} v_{1}=D_{1} v_{2} ; \mu=12 \sqrt{1-v_{1} v_{2}},
\end{aligned}
$$

where $C$ - a characteristic value of one of the coefficients $C_{1} ; \rho$ and $\bar{t}$-dimensionless radial coordinate and dimensionless time; $($ - stress function; $\theta$ - function of the angles of rotation of shell's generating curve; $\varphi$ - stress function; $w$ - deflection function; $q$ - projection of the distributed loading on the $z$-axis coordinate; $d f(\rho)$ - generatrix function for shell of revolution; $r$ - radial coordinate; $t$ - time; $g$ - acceleration of gravity; $h$ - thickness of the shell; $E_{i}$ - elastic modulus, $D_{i}$ - bending stiffness of shell, $v_{i}$ - Poisson ratio. Here, the value of the index $i=1$ corresponds to the radial direction, $i=2-$ corresponds to the circumferential direction.

Load acting on the shell can be represented as the sum of the static and dynamic component:

$$
q(r, t)=q_{0}(r)+q_{1}(r, t)
$$


We represent the functions $\Phi$ and $\theta$ as a sum of components defining the initial state of the shell $\left(\Phi_{0}\right.$ and $\theta_{0}$ ) and components describing deviation of the system in the process of oscillation ( $\bar{\Phi}$ and $\bar{\theta}$ ):

$$
\Phi=\Phi_{0}+\bar{\Phi} ; \theta=\theta_{0}+\bar{\theta}
$$

Write the function the influence of inertial forces in form:

$$
\alpha=\alpha(\rho, \bar{t})=\frac{\sqrt{\mu}}{h \rho} \int_{\rho_{0}}^{\rho} \rho \frac{\partial^{2} w(\rho, \bar{t})}{\partial \bar{t}^{2}} d \rho
$$

Equations (5) linearize the small deviations of the system relation to the initial equilibrium state $[02,06,07]$. The system of equations is separated into system, which defines the initial deformed state the shell and the system describing the oscillations of a shell.

The first system of equations is similar to the one in $[01,02]$. Second, after linearization written as:

$$
\begin{aligned}
& \rho \frac{\partial}{\partial \rho}\left(\frac{\bar{C}_{12}}{\rho} \bar{\Phi}-\bar{C}_{1} \frac{\partial \bar{\Phi}}{\partial \rho}\right)+\frac{\left(\bar{C}_{2}+\bar{C}_{12}\right)}{\rho} \bar{\Phi}-\left(\bar{C}_{1}+\bar{C}_{12}\right) \frac{\partial \bar{\Phi}}{\partial \rho}+K \bar{\theta}+\theta_{0} \bar{\theta}=0 \\
- & \frac{\partial}{\partial \rho}\left(\rho \bar{D}_{1} \frac{\partial \bar{\theta}}{\partial \rho}+\bar{D}_{12} \bar{\theta}\right)+\bar{D}_{12} \frac{\partial \bar{\theta}}{\partial \rho}+\frac{\bar{D}_{2}}{\rho} \bar{\theta}-K \bar{\Phi}-\Phi_{0} \bar{\theta}-\bar{\Phi} \theta_{0}-\bar{\alpha} \rho=q_{1}(\rho, \bar{t}) \\
& \rho \frac{\partial}{\partial \rho}\left(\frac{1}{\rho} \frac{\partial}{\partial \rho}(\bar{\alpha} \rho)\right)=\omega^{2} \rho \bar{\theta}
\end{aligned}
$$

The last of the equations in (5) is obtained by double differentiation on $\rho$ and replacement $\frac{\partial^{3} w(\rho, \bar{t})}{\partial \tilde{t}^{2} \partial \rho}=\frac{\partial^{2} \bar{\theta}}{\partial \hat{t}^{2}}$ according to (2).

Support contour is modeled as the elastic fixing, which is a uniform representation of the different types of supports.

The boundary conditions of the problem are taken in the following form:

For the elastic fixing with elasticity coefficients of the support contour $m$ and $n$ :

$$
n \Phi-\rho_{1} \bar{C}_{1} \frac{\partial \Phi}{\partial \rho}=0, m \theta-\rho_{1} \bar{D}_{1} \frac{\partial \theta}{\partial \rho}=0, \alpha=0
$$

On the top closed shell as:

$$
\theta=0, \Phi=0, \alpha=0
$$

On the free upper edge of the shell, which is not closed at the top as:

$$
\Phi=-\bar{N}_{r} C \sqrt{\mu} \rho_{0}, \bar{D}_{1} \frac{d \theta}{d \rho}+\bar{D}_{12} \frac{\theta}{\rho_{0}}=-\frac{\bar{M}_{r} C \mu}{h}, \alpha=0
$$

where $\bar{N}_{r}, \bar{M}_{r}$ is the static load applied to the upper edge of the contour in form evenly distributed force and evenly distributed bending moment, respectively.

We assume that the shell is affected by a harmonic disturbance load:

$$
q_{1}(\rho, \bar{t})=q_{A}(\rho) \cdot \cos (\omega \cdot \bar{t})
$$

where $\omega$ is a frequency of disturbing force, $q_{A}(\rho)$ is a function describing the distribution of the load amplitude values along the generatrix of shell.
We assume that the oscillations of the shell are steady, with a frequency equal to the frequency of the disturbing load:

$$
\begin{aligned}
& \bar{\Phi}(\rho, \bar{t})=\Phi(\rho) \cdot \cos (\omega \cdot \bar{t}) ; \\
& \bar{\theta}(\rho, \bar{t})=\theta(\rho) \cdot \cos (\omega \cdot \bar{t}) ; \bar{\alpha}(\rho, \bar{t})=\alpha(\rho) \cdot \cos (\omega \cdot \bar{t})
\end{aligned}
$$

where $\Phi(\rho), \theta(\rho), \alpha(\rho)$ is the amplitude values of the corresponding functions.

Dynamic analysis of the shell is carried out in two stages. First, the problem of determining the initial stress-strain state from the static component of the acting loads. The finite-element methodology of solving this problem is described in [01, 02]. Further, the resulting values $\Phi_{0}, \theta_{0}$ are used to solve the system of equations (6) describing the oscillations of the shell.

Construction of a finite element is carried out by the Galerkin method [01-04]. The mixed form of the system of equations in combination with the Galerkin method in the weak form allows our to choose the shape function of the finite element in the class of linear functions:

$$
\begin{aligned}
& \Phi(\rho)=\Phi_{1} N_{1}(\rho)+\Phi_{2} N_{2}(\rho) ; \\
& \theta(\rho)=\theta_{1} N_{1}(\rho)+\theta_{2} N_{2}(\rho) ; \\
& \alpha(\rho)=\alpha_{1} N_{1}(\rho)+\alpha_{2} N_{2}(\rho)
\end{aligned}
$$

where $\Phi_{\mathrm{i}}, \theta_{\mathrm{i}}, \alpha_{\mathrm{i}}$ are the nodal values of the functions $\Phi, \theta$ and $\alpha ; N_{i}-$ are linear shape functions.

After integrating the equation of the Galerkin method, we obtain a system of equations describing the oscillations of the shell in the form:

$$
\left([S]-\omega^{2}[M]-\left[G_{0}\left(\left\{q_{0}\right\}\right)\right]\right)\{q\}=\{F\}
$$

Where $[S]$ is the matrix of element (stiffness matrix); $[\mathrm{M}]$ is the mass matrix element; $\{\mathrm{q}\}=\left[\Phi_{1}, \theta_{1}, \alpha_{1}, \Phi_{2}, \theta_{2}, \alpha_{2}\right]$ is the vector of nodal parameters; $\left[\mathrm{G}_{0}\left(\left\{q_{0}\right\}\right)\right]$ is the matrix containing components that depend on the values of the node parameters calculated for the initial stress-strain state $\left\{q_{0}^{\star}\right\} ; \omega$ is the frequency of the disturbing force; $\chi, \eta$ is the $\rho$ coordinates of element's nodes;

$$
\begin{aligned}
& {[S]=\left[\begin{array}{cccccc}
e_{1}+e_{2}+\chi \bar{C}_{12} & e_{3} & 0 & -e_{1}+\frac{e_{2}}{2} & e_{4} & 0 \\
-e_{3} & e_{5}+e_{6}-\chi \bar{D}_{12} & -e_{14} & -e_{4} & -e_{5}+\frac{e_{6}}{2} & -e_{15} \\
0 & 0 & e_{12} & 0 & 0 & e_{10} \\
-e_{7}+\frac{e_{2}}{2} & e_{4} & 0 & e_{7}+e_{2}-\eta \bar{C}_{12} & e_{8} & 0 \\
-e_{4} & -e_{9}+\frac{e_{6}}{2} & -e_{15} & -e_{8} & e_{9}+e_{6}+\eta \bar{D}_{12} & -e_{16} \\
0 & 0 & e_{11} & 0 & 0 & e_{13}
\end{array}\right]} \\
& e_{1}=\frac{\left(\eta^{2}+\eta \chi+4 \chi^{2}\right)}{6(\eta-\chi)} \bar{C}_{1} ; e_{2}=\frac{(\eta-\chi)}{3} \bar{C}_{2} ; e_{3}=\frac{\left(\eta^{2}+2 \eta \chi-3 \chi^{2}\right)}{12} K ; \\
& e_{4}=\frac{\left(\eta^{2}-\chi^{2}\right)}{12} K ; e_{5}=\frac{\left(\eta^{2}+\eta \chi+4 \chi^{2}\right)}{6(\eta-\chi)} \bar{D}_{1} ; e_{6}=\frac{(\eta-\chi)}{3} \bar{D}_{2} ; \\
& e_{7}=\frac{\left(4 \eta^{2}+\eta \chi+\chi^{2}\right)}{6(\eta-\chi)} \bar{C}_{1} ; e_{8}=\frac{\left(3 \eta^{2}-2 \eta \chi-\chi^{2}\right)}{12} K ;
\end{aligned}
$$




$$
\begin{aligned}
& e_{9}=\frac{\left(4 \eta^{2}+\eta \chi+\chi^{2}\right)}{6(\eta-\chi)} \bar{D}_{1} ; e_{10}=\frac{\chi(\eta+\chi)}{2(\eta-\chi)} ; e_{11}=\frac{\eta(\eta+\chi)}{2(\chi-\eta)} \\
& e_{12}=\frac{\eta^{2}-3 \eta \chi+4 \chi^{2}}{2(\chi-\eta)} ; e_{13}=\frac{4 \eta^{2}-3 \eta \chi+\chi^{2}}{2(\chi-\eta)} ; \\
& e_{14}=\frac{\eta^{3}+2 \eta^{2} \chi+3 \eta \chi^{2}-6 \chi^{3}}{30} ; e_{15}=\frac{3 \eta^{3}+\eta^{2} \chi-\eta \chi^{2}-3 \chi^{3}}{60} ;
\end{aligned}
$$$$
[M]=\left[\begin{array}{cccccc}
0 & 0 & 0 & 0 & 0 & 0 \\
0 & 0 & 0 & 0 & 0 & 0 \\
0 & \frac{\eta^{3}+2 \eta^{2} \chi+3 \eta \chi^{2}-6 \chi^{3}}{30} & 0 & 0 & \frac{3 \eta^{3}+\eta^{2} \chi-\eta \chi^{2}-3 \chi^{3}}{60} & 0 \\
0 & 0 & 0 & 0 & 0 & 0 \\
0 & 0 & 0 & 0 & 0 & 0 \\
0 & \frac{3 \eta^{3}+\eta^{2} \chi-\eta \chi^{2}-3 \chi^{3}}{60} & 0 & 0 & \frac{6 \eta^{3}-3 \eta^{2} \chi-2 \eta \chi^{2}-\chi^{3}}{30} & 0
\end{array}\right]
$$$$
\left[G_{0}\left(\left\{g_{0}\right)\right]=\left[\begin{array}{cccccc}
0 & \frac{3 e_{17} \theta_{1}^{0}+e_{20} \theta_{2}^{0}}{60} & 0 & 0 & \frac{e_{20} \theta_{1}^{0}+e_{19} \theta_{2}^{0}}{60} & 0 \\
-\frac{3 e_{17} \theta_{1}^{0}+e_{20} \theta_{2}^{0}}{60} & -\frac{3 e_{17} \Phi_{1}^{0}+e_{20} \Phi_{2}^{0}}{60} & 0 & -\frac{e_{20} \theta_{1}^{0}+e_{19} \theta_{2}^{0}}{60} & -\frac{e_{20} \Phi_{1}^{0}+e_{19} \Phi_{2}^{0}}{60} & 0 \\
0 & 0 & 0 & 0 & 0 & 0 \\
0 & \frac{e_{20} \theta_{1}^{0}+e_{19} \theta_{2}^{0}}{60} & 0 & 0 & \frac{e_{19} \theta_{1}^{0}+3 e_{18} \theta_{2}^{0}}{60} & 0 \\
-\frac{e_{20} \theta_{1}^{0}+e_{19} \theta_{2}^{0}}{60} & -\frac{e_{20} \Phi_{1}^{0}+e_{19} \Phi_{2}^{0}}{60} & 0 & -\frac{e_{19} \theta_{1}^{0}+3 e_{18} \theta_{2}^{0}}{60} & -\frac{e_{19} \Phi_{1}^{0}+3 e_{18} \Phi_{2}^{0}}{60} & 0 \\
0 & 0 & 0 & 0 & 0 & 0
\end{array}\right]\right.
$$$$
e_{16}=\frac{6 \eta^{3}-3 \eta^{2} \chi-2 \eta \chi^{2}-\chi^{3}}{30} ; e_{17}=4 \chi^{2}-3 \chi \eta-\eta^{2}
$$$$
e_{18}=\chi^{2}+3 \chi \eta-4 \eta^{2} ; e_{19}=2 \chi^{2}+\chi \eta-3 \eta^{2} ; e_{20}=3 \chi^{2}-\chi \eta-2 \eta^{2} ;
$$$$
\vec{F}=\left\{\begin{array}{c}
\frac{q_{A} h C \sqrt{\mu}}{120}\left(-8 \chi^{4}+7 \eta^{2} \chi^{2}+7 \eta \chi^{3}-3 \eta^{3} \chi-3 \eta^{4}\right)-\frac{s_{i}}{6}\left(\eta^{2}+\eta \chi-2 \chi^{2}\right) \\
0 \\
0 \\
\frac{q_{A} h C \sqrt{\mu}}{120}\left(-7 \chi^{4}+23 \eta^{2} \chi^{2}-7 \eta \chi^{3}-7 \eta^{3} \chi-12 \eta^{4}\right)-\frac{s_{i}}{6}\left(2 \eta^{2}-\eta \chi-\chi^{2}\right) \\
0
\end{array}\right\}
$$

Here $q_{A}$ is the amplitude values of the uniformly distributed load acting on the element, $s_{i}$ is the value of the load integral, calculated for all elements, starting from the element closest to the center of the shell, to the current element ( $i$-th element):

$$
s_{i}=\sum_{j=1}^{i-1} \frac{\sqrt{\mu}}{E} \int_{\chi_{j}}^{\eta_{j}} q_{A}(\rho) \rho d \rho
$$

The values of $s_{i}$ are calculated for all elements before the procedure for assembling the generalized matrices and vectors of the problem.

The resulting finite-element relations have a simple structure, and the calculation of the matrices and vectors of the system (13) does not require the use of numerical integration, which positively affects the accuracy of the results obtained.

The boundary conditions, consisting in equating to zero the values of one or several functions, are implemented by the usual technique for the finite element method.

The boundary conditions (7) for the functions $\Phi$ and $\theta$ on the support contour are taken into account by adding the values $\eta\left(\bar{C}_{1}-n\right)$ (for function $(\Phi)$ and $\eta\left(\bar{D}_{1}-m\right)$ (for function $\theta$ ) to the corresponding diagonal elements of the generalized matrix.

For the upper free edge of a non-closed shell, the boundary conditions (9) for the functions $\Phi$ and $\theta$ are taken into account by adding the matrix $\left[\mathrm{S}_{\mathrm{bc}}\right]$ and the vector $\left\{F_{b c}\right\}$ of boundary conditions to the matrix [S] and to the vector $\{\mathrm{F}\}$, respectively. The matrix $\left[\mathrm{S}_{\mathrm{bc}}\right]$ and the vector $\left\{F_{b c}\right\}$ have the following form:

$$
\begin{aligned}
& {\left[S_{b c}\right]=\left[\begin{array}{cccc}
1-e_{3} & -e_{4} & -e_{1} \bar{C}_{1}-\frac{\eta-\chi}{6} \bar{C}_{2} & -\frac{K}{12}\left(\eta^{2}-\chi^{2}\right) \\
0 & 0 & 0 & 0 \\
0 & 0 & 0 & 0 \\
0 & 0 & 0 & 0
\end{array}\right]} \\
& \left\{F_{b c}\right\}=\left\{-\bar{N}_{r} C \sqrt{\mu} \chi \quad-\frac{\bar{M}_{r} C \mu}{h} \quad 0 \quad 0 \quad\right\}^{\mathrm{T}}
\end{aligned}
$$

Assembling generalized matrices and vectors of the problem is usually carried out for the finite element method.

The system of equations obtained after assembling and taking into account the boundary conditions is solved by the Gauss method or by another suitable method for solving systems of linear algebraic equations.

\section{CHECKING THE RESULTS}

To establish the convergence of finite-element method plotted $\mathrm{Nr}$ force change on the partition of the shell with a different number of elements in radial direction. When the number of elements is more than 15-20, the difference between the obtained values is less than $0.1 \%$.

To cheking of the results obtained using the developed methodology, a test calculation of the isotropic shell of a spherical shape was carried out according to the undeformed scheme. These results were compared with the solution obtained by the finite element method in displacements formulation using the SCAD program. The difference in values was not more than $5 \%$.

The correctness of the results obtained is confirmed by the amplitude-frequency characteristic of the shell (see Figure 1). The frequencies at which resonance is observed are very close to the values of the eigenfrequency of the oscillations of the shell obtained by the methodology [02]: 13477, 24261, and $51948 \mathrm{~Hz}$.

\section{INVESTIGATION OF DYNAMIC RESPONSE OF SHELL}

The influence of the static component of the load on the shell $p_{0}=h c \sqrt{\mu}\left(\frac{a}{h} \sqrt[4]{\mu}\right)^{2} q_{0}$, which determines the initial stressstrain state of the shell, on the amplitude values of forces and displacements is investigated.

We considered a top closed shallow shell of revolution of spherical shape with rigid fixed support contour made of isotropic material. Geometrical parameters of the shell: $\quad \frac{f_{\max }}{a} \sqrt[4]{\mu}=0,15, \frac{a}{h} \sqrt[4]{\mu}=50$. 
Here $f_{\max }$ is the rising height of the shell, and $a$ is the radius of the base.

The amplitude value of the dynamic component of the load was assumed to be unity.

Based on the results of the performed calculations graphs are constructed for the changing of the function $\Phi, \theta$ and the deflection $w$ of shell in radial direction (see Figures 2-4). For convenience of comparison, the values of the functions are given to a range of values from 0 to 1 . The dashed lines show graphs calculated from the linear theory. The graphs in Figures 2-4 show that with a constant dynamic load, with an increase in the static component of the load, the amplitude values of functions and displacements increase during the process of oscillation of the shell. This effect is explained by the nonlinearity of the shell deformation model.

In Figure 5 shows the amplitude-frequency response of the shell for various values of the static component of the load, which determines the initial stress-strain state. As can be seen from the figure, when the static load changes, the graphs of the amplitude-frequency response of the shell change with a shift in the resonance frequencies toward a decrease.

\section{CONCLUSIONS}

The developed finite element makes it possible to realize the effective methodology of dynamic analysis of orthotropic flexible shells of revolution with allowance for the initial deformed state.

The carried out researches of the influence of the shell's initial stress-strain state on the amplitude values of the forces and displacements that appear in the process of forced vibrations demonstrate the need to take this into account when performing a dynamic analysis of non-linear shallow shells.

\section{REFERENCES}

1. Stupishin, L.U., \& Nikitin, K.E. (2014). Mixed finite element for geometrically nonlinear orthotropic shallow shells of revolution. Applied Mechanics and Materials, 919-921, 1299-1302.

2. Stupishin, L.U., \& Nikitin, K.E. (2014). Numerical research methodology of free oscillations of geometrically nonlinear shell using the mixed finite element method. Applied Mechanics and Materials, 580-583, 3017-3020.

3. Stupishin, L.U., \& Kolesnikov, A.G. (2014). Geometric Nonlinear Orthotropic Shallow Shells Investigation. Applied Mechanics and Materials, 501-504, 766-769.

4. Fletcher, C.A.J. (1984). Computational Galerkin methods. New York: Springer-Verlag Inc..

5. Strang, G., \& Fix, G. (2008). An Analysis of the Finite Element Method. Wellesley-Cambridge Press.
6. Valishvili, N.V. (1976). Metody rascheta obolochek vrashcheniya na ETsVM. Moscow: Mashinostroenie Publ..

7. Volmir, A.C. (1972). Nelineynaya dinamika plastinok i obolochek. Moscow: Nauka Publ..

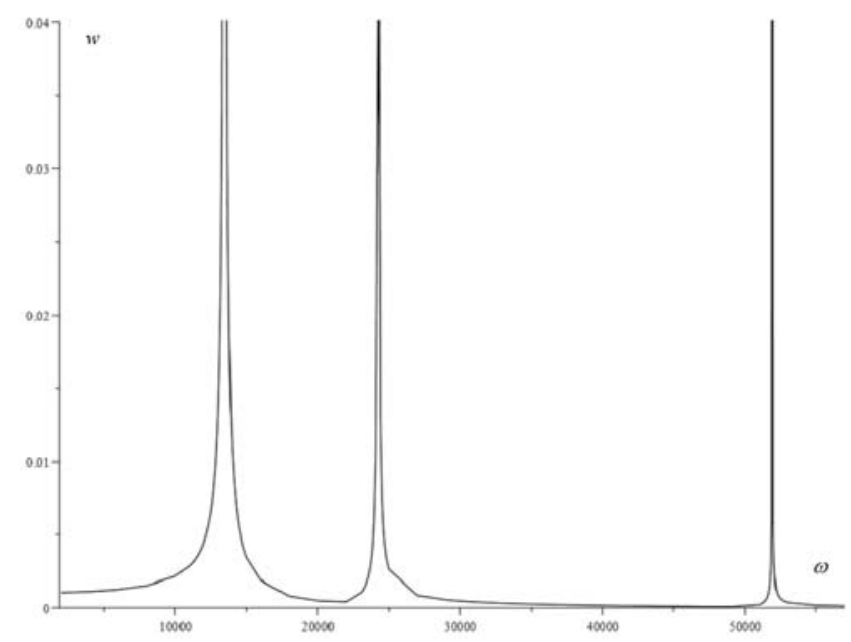

Figure 1: Amplitude frequency response of the test shell

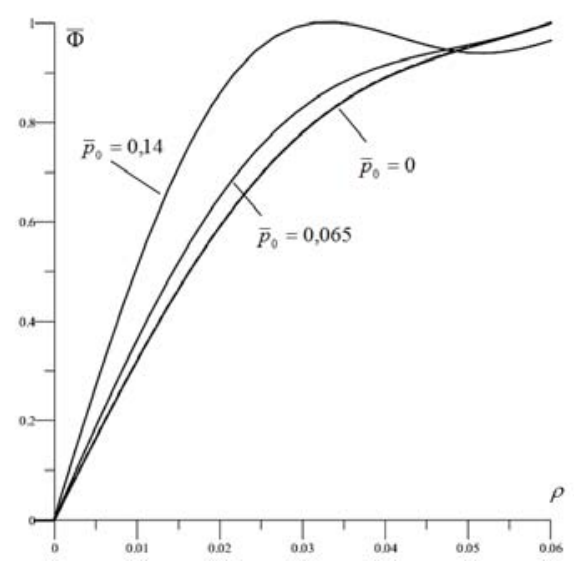

Figure 2: Change of the function $\Phi$ in radial direction for different values of the static component of the load on the shell 


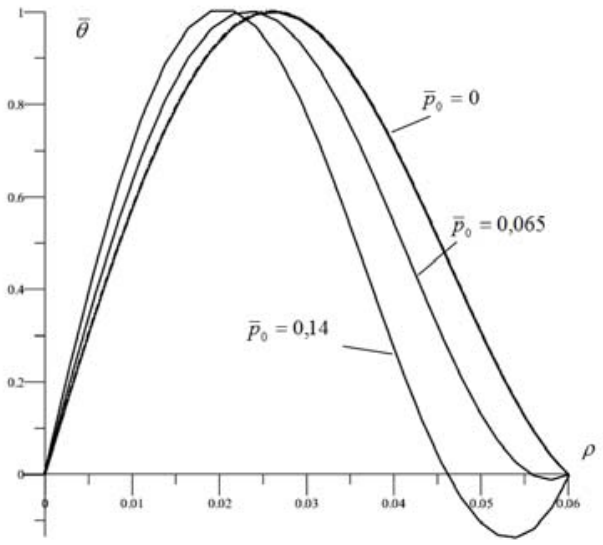

Figure 3: Change of the function $\theta$ in radial direction for different values of the static component of the load on the shell

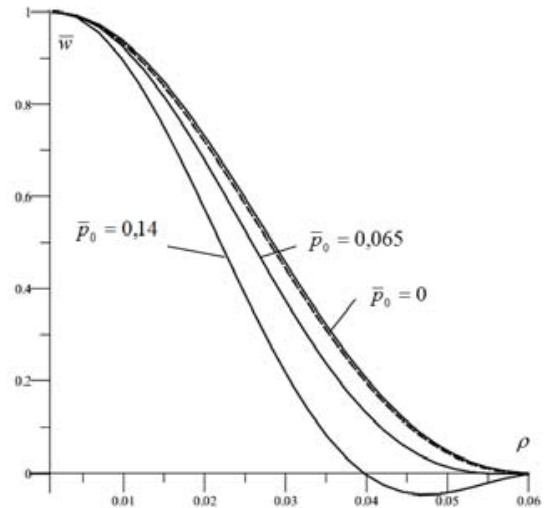

Figure 4: Change of the deflection $w$ of the shell in radial direction for different values of the static component of the load on the shell

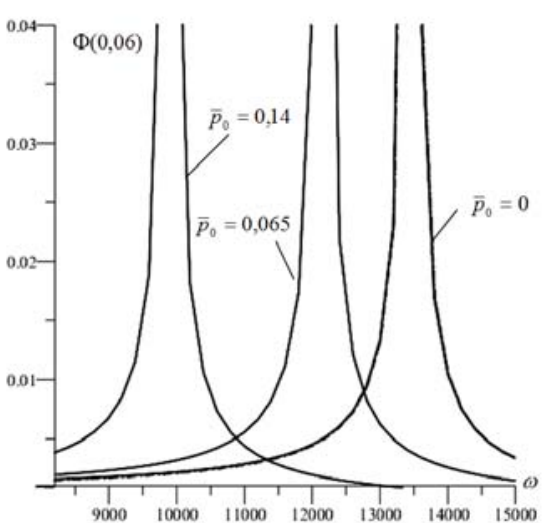

Figure 5: Amplitude-frequency response of the shell for different values of the static component of the load 